\title{
Necessary and Sufficient Conditions for Reachability on a Simplex
}

\author{
Bartek Roszak and Mireille E. Broucke \\ Edward S. Rogers Sr. Department of Electrical \\ and Computer Engineering \\ University of Toronto \\ 10 King's College, M5S 3G4, Toronto Ontario, \\ Canada \\ \{bartek, broucke\}@control.utoronto.ca
}

\begin{abstract}
The reachability problem has received significant attention in the hybrid control literature with many questions still left unanswered. In this paper we solve the general problem of reaching a set of facets of an n-dimensional simplex in finite time, for a system evolving with linear affine dynamics. Necessary and sufficient conditions are presented in the form of inequalities on the vertices of the simplex, and a linear affine controller is constructed that solves the reachability problem.
\end{abstract}

\section{INTRODUCTION}

In this paper we present reachability results for linear affine systems defined on a polytope or simplex. We particularly consider the synthesis of controllers that achieve a particular reachability specification on a simplex and we develop necessary and sufficient conditions for the existence of such a controller. The work of this paper is primarily motivated by previous results on reachability and controllability on polytopes presented by Habets and van Schuppen [8], and is related to work on invariance of polyhedral sets as presented in the survey paper by Blanchini $[5$, pp. 17541756].

Our results have implications for reachability analysis in hybrid system theory. A hybrid system combines the continuous model of several systems or subsystems with discrete transitions that occur in between or within them. In recent years, the interest in hybrid systems has grown considerably and various general results have been presented in proceedings; for example [1] and [11]. Our interest lies specifically with linear affine systems studied by Habets and van Schuppen [8], and introduced by Sontag [13], [14], [15].

In the literature, several interesting publications are related to our work. For instance, Lee and Arapostathis [10] investigated global controllability of piecewise-linear affine hypersurface systems, while Veliov and Krastanov [17] studied local controllability of a system which is linear on two half-spaces. Other related work on invariant polyhedral sets of linear systems have been studied by Vassilaki and Bitsoris [16] and Castelan and Hennet [6]. The survey paper by Blanchini [5] on set invariance in control provides many other related references. The problem of reaching a particular facet of an $n$-dimensional polytope has been extensively studied by Habets and van Schuppen [7], [8]. The present paper is highly motivated by their results. However, we relax a key condition in [8], allowing to find necessary and sufficient conditions for a more general problem of steering a state of the system to a set of facets of a simplex. Moreover, we show through examples that even though the conditions for reachability of a facet in [8] are infeasible, we may still be able to find a continuous state feedback which guarantees the control objective.

The paper is organized as follows. The terminology, adopted from [8], and the problem statement is presented next. Section II contains background work related to the paper. Next, necessary and sufficient conditions for the existence of a continuous state feedback control which achieves the control objectives is presented in Section III. An algorithm for constructing a continuous controller is presented in Section IV. Finally, several examples are given in Section V. Concluding remarks follow, summarizing the results and outlining our future directions of research.

\section{A. Terminology}

The convex hull of a set $P \subset \mathbb{R}^{n}$ is the set of all convex combinations of points in $P$. An $n$-dimensional polytope is a set $P_{n} \subset \mathbb{R}^{n}$, defined as the convex hull of a set of points $V:=\left\{v_{1}, \ldots, v_{k} \mid k \geq n+1\right\}$, where $v_{1}, \ldots, v_{k}$ are chosen such that there does not exist a hyperplane of $\mathbb{R}^{n}$ containing all points of $V$. A vertex of a set $P$ is any point of $P$ which cannot be written as a convex combination of any other distinct points of $P$. The set of vertices of $P$ will be denoted as $V$.

Given a set of $m \in \mathbb{N}$ vectors $\left\{x_{1}, \ldots, x_{m} \mid x_{i} \in \mathbb{R}^{n}\right\}$, $\sum_{i=1}^{m} \alpha_{i} x_{i}$ is called an affine combination if $\sum_{i=1}^{m} \alpha_{i}=1$. The affine hull of a set $P$ is the set of all affine combinations of points in $P$, denoted aff $(P)$. A set of $q+1$ points $\left\{v_{1}, \ldots, v_{q+1}\right\}$ in $\mathbb{R}^{n}$ is said to be affinely independent if af $f\left(\left\{v_{1}, \ldots, v_{q+1}\right\}\right)$ is $q$-dimensional. If a set of points $\left\{v_{1}, \ldots, v_{n+1}\right\}$ is affinely independent, then its convex hull is called an $n$-dimensional simplex, denoted $S_{n}$. In this paper, whenever we say polytope (simplex), we mean an $n$ dimensional polytope (simplex).

For an n-dimensional polytope, there exist $j \geq n+1$ distinct unit length normals $h_{1}, \ldots, h_{j} \in \mathbb{R}^{n}$ and $j$ non-zero reals $\alpha_{1}, \ldots, \alpha_{j} \in \mathbb{R}$ such that the polytope can be written 
as an intersection of $j(n-1)$-dimensional hyperplanes:

$$
P_{n}:=\left\{x \in \mathbb{R}^{n} \mid h_{i} \cdot x \leq \alpha_{i}, \quad i=1,2, \ldots, j\right\} .
$$

By convention the normal vectors $h_{i}$ point out of $P_{n}$. A facet of a polytope $P_{n}$ is an $(n-1)$-dimensional intersection of the polytope with a supporting hyperplane:

$$
F_{i}:=\left\{x \in \mathbb{R}^{n} \mid h_{i} \cdot x=\alpha_{i}\right\} \cap P_{n} \quad i=1, \ldots, j .
$$

If $P_{n}$ is a simplex, then we use the convention that $F_{i}$ is the convex hull of $\left\{v_{1}, \ldots, v_{i-1}, v_{i+1}, \ldots, v_{n+1}\right\}$ where $v_{j} \in V$.

Lastly, we define a multi-input linear affine system as

$$
\dot{x}=A x+B u+a,
$$

where $x \in \mathbb{R}^{n}, u \in \mathbb{R}^{m}$, and $a \in \mathbb{R}^{n}$ and a linear affine state feedback function of the form:

$$
u=F x+g,
$$

where $F \in \mathbb{R}^{m \times n}$ and $g \in \mathbb{R}^{m}$. Notice that with this state feedback the closed loop system is of affine form

$$
\dot{x}=(A+B F) x+(B g+a)=\tilde{A} x+\tilde{a} .
$$

\section{B. Problem Statement}

Consider a linear affine system $\dot{x}=\tilde{A} x+\tilde{a}$ defined on a polytope $P_{n}$. A facet $F_{i}$ of the polytope $P_{n}$ is called restricted or invariant if no trajectory of the system exits through the facet. (A facet which is not restricted is called unrestricted). Let $I$ be a given set of indices of the facets to be made restricted by proper choice of control input for the system (3). We assume at least one facet $F_{1}$ is not restricted, i.e. $1 \notin I$. Also, for each $v \in V$, let $I_{v}=\{k \mid k \in I, v \in$ $\left.F_{k}\right\}$. We consider the following problem.

Problem 1.1: Let $P_{n}$ be an $n$-dimensional polytope with a set of facets $F_{j}, j \in I$, with normal vectors $h_{j}$. For the system (3), construct a continuous feedback $u=f(x), f$ : $P_{n} \rightarrow \mathbb{R}^{m}$ such that for each initial condition $x_{0} \in P_{n}$ there exist a time $t_{0} \geq 0$ and an $\epsilon>0$ such that

1) $\forall t \in\left[0, t_{0}\right], x(t) \in P_{n}$,

2) $x\left(t_{0}\right) \in F_{k}$, for some $k \notin I$

3) $\forall t \in\left(t_{0}, t_{0}+\epsilon\right), x(t) \notin P_{n}$.

The problem states that we must design $u$ to enforce all controlled trajectories originating inside $P_{n}$ to escape $P_{n}$ through some $F_{k}$, which is not invariant (notice that nothing is said about the trajectory for $\left.t>\left(t_{0}+\epsilon\right)\right)$. In contrast with [8], we do not try to restrict $n$ facets, but rather an arbitrary number. Also, we drop the restriction that $t_{0}$ must be the first time at which the state reaches the exit facet. Thus, the current problem is a generalization of the one in [8]. The implications of the extra restriction in [8] will be highlighted in the sequel. Since any n-dimensional polytope can be partitioned into simplices via triangulation, the focus of this paper will be on solving Problem 1.1 with $P_{n}$ assumed to be a simplex $S_{n}$. For an excellent overview of triangulation and its complexities, the reader is referred to [8] and the references therein.

\section{BACKGROUND}

In this section, we give background results needed for the remainder of the paper. First, we present results from convex analysis concerning separating hyperplanes that will be used for our main results. Second, two elements from [8] are reviewed. First, we show how a linear affine controller can be constructed using only the input values at the vertices. Second, we give conditions for preventing trajectories originating inside a polytope from leaving via a particular facet.

\section{A. Convex Sets and Separating Hyperplanes}

This subsection presents two important results related to convex sets and separating hyperplanes. These will be used in a proof of a key result in Section III.

Definition 2.1 ([12] pg.95): Let $C_{1}$ and $C_{2}$ be non-empty sets in $\mathbb{R}^{n}$. A hyperplane $H$ is said to separate $C_{1}$ and $C_{2}$ if $C_{1}$ is contained in one of the closed half-spaces associated with $H$, while $C_{2}$ lies in the opposite closed half-space. Let $B:=\{x \mid\|x\| \leq 1\}$. If there exists $\epsilon>0$ such that $C_{1}+\epsilon B$ is contained in one of the open half-spaces associated with $H$ and $C_{2}+\epsilon B$ is contained in the opposite open half-space, then $C_{1}$ and $C_{2}$ are separated strongly.

The following theorem gives a condition for the existence of a hyperplane which separates two non-empty convex sets.

Theorem 2.1 ([12] pg.98): Let $C_{1}$ and $C_{2}$ be non-empty convex sets in $\mathbb{R}^{n}$. In order that there exist a hyperplane $H$ separating $C_{1}$ and $C_{2}$ strongly, it is necessary and sufficient that

$$
\inf \left\{\left\|x_{1}-x_{2}\right\| \mid x_{1} \in C_{1}, x_{2} \in C_{2}\right\}>0 .
$$

Notice that if $C_{1}$ and $C_{2}$ are convex and compact, and $C_{1} \cap C_{2}=\emptyset$, then (5) holds, implying that such an $H$ exists.

\section{B. Linear Affine Systems defined on Polytopes}

Next we review several results from [8]. The main ideas are as follows. Given a simplex $S_{n}$, we would like to impose certain conditions, called invariance conditions and flow conditions at the vertices of the simplex, which guarantee that trajectories may not leave from the restricted facets, but may leave from the unrestricted facets. The invariance conditions, introduced in [8], dictate that the vector field may not point "out" of a restricted facet. Lemma 2.5 shows that the invariance conditions indeed guarantee that no trajectory can exit the restricted facet. The flow conditions are a new element contributed in this paper. A flow condition imposes that the vector field points in a particular direction with respect to a given vector $\xi \in \mathbb{R}^{n}$, or equivalently, points along a particular side of a hyperplane with normal vector $\xi$. In Section III we will show that the flow conditions provide the required additional element to solve the more general reachability problem of this paper. Lemma 2.2 says that an invariance (or flow) condition on a facet can be acheived simply by imposing the invariance or flow condition on the vertices of the simplex. Thus, the procedure is to write invariance and flow conditions at the vertices only and solve them for the control values at the vertices. Lemma 2.1 shows 
that once those control values at the vertices are obtained, one can construct a linear affine feedback control defined on the entire simplex such that the closed-loop system achieves the design objective.

The first result of this subsection shows how a linear affine controller can be constructed for the system (3) defined on a simplex, from the knowledge of the input values at the vertices. The proof parallels the result of [8] and is presented for completeness.

Lemma 2.1: Consider two sets of points $\left\{v_{1}, \ldots, v_{n+1}\right\}$, $v_{j} \in \mathbb{R}^{n}$ and $\left\{u_{1}, \ldots, u_{n+1}\right\}, u_{j} \in \mathbb{R}^{m}$. Suppose the $v_{j}$ 's are affinely independent. Then there exists a unique matrix $F \in \mathbb{R}^{m \times n}$ and a unique vector $g \in \mathbb{R}^{m}$ such that for each $v_{j}, u_{j}=F v_{j}+g$.

Proof: We want to show there exists a unique matrix $F$ and a unique vector $g$ such that, in matrix form,

$$
\left[\begin{array}{ll}
v_{1}^{T} & 1 \\
v_{2}^{T} & 1 \\
\vdots & \\
v_{n+1}^{T} & 1
\end{array}\right]\left[\begin{array}{l}
F^{T} \\
g^{T}
\end{array}\right]=\left[\begin{array}{l}
u_{1}^{T} \\
u_{2}^{T} \\
\vdots \\
u_{n+1}^{T}
\end{array}\right] .
$$

If the $(n+1) \times(n+1)$ left-hand matrix is full rank, then multiplying by its inverse yields the unique solutions $F$ and $g$. However,

$\operatorname{rank}\left[\begin{array}{ll}v_{1}^{T} & 1 \\ v_{2}^{T} & 1 \\ \vdots & \\ v_{n+1}^{T} & 1\end{array}\right]=1+\operatorname{rank}\left[\begin{array}{l}v_{2}^{T}-v_{1}^{T} \\ v_{3}^{T}-v_{1}^{T} \\ \vdots \\ v_{n+1}^{T}-v_{1}^{T}\end{array}\right]=1+n$.

The last equality follows since the points $\left\{v_{1}, \ldots, v_{n+1}\right\}$ are affinely independent if and only if the affine hull of $\left\{v_{1}, \ldots, v_{n+1}\right\}$ is $\mathrm{n}$-dimensional, i.e. the vectors $\left\{v_{2}-\right.$ $\left.v_{1}, \ldots, v_{n+1}-v_{1}\right\}$ must be linearly independent.

Since a simplex is the convex hull of $n+1$ affinely independent points, it is now clear that if we enforce input values $u_{1}, \ldots, u_{n+1}$ at the $n+1$ vertices of the simplex, then we can also construct a corresponding linear affine controller $u=F x+g$.

The next preliminary result, proved in [8], shows that once a controller is specified on the vertices of $S_{n}$, its invariance properties can be extended to every point in $S_{n}$. An alternate interpretation, which is new in our work, is the following: if a flow condition with respect to a hyperplane holds for a linear affine vector field at the vertices of $S_{n}$, then that flow condition holds for all points in $S_{n}$. Intuitively this statement means that as long as a vector field has a particular direction of flow with respect to a hyperplane with normal vector $\xi$, on all the vertices of $S_{n}$, then the vector field also has that direction of flow on all points of $S_{n}$.

Lemma 2.2: Consider system (3) defined on a polytope $P_{n}$ with vertices $V=\left\{v_{1}, \ldots, v_{k}\right\}$. Given $\xi \in \mathbb{R}^{n}, \xi \neq 0$, for all $x \in P_{n}$ there exists an input $u \in \mathbb{R}^{m}$ such that

$$
\xi \cdot(A x+B u+a)<0
$$

if and only if for all $v_{j} \in V$ there exists $u_{j} \in \mathbb{R}^{n}$ such that

$$
\xi \cdot\left(A v_{j}+B u_{j}+a\right)<0 .
$$

Note that in the previous Lemma $<$ can be replaced with any of $\{\leq,>, \geq,=\}$. In particular, we use $\leq$ when dealing with invariance properties.

The next two preliminary results are needed in order to prove Lemma 2.5, which describes when a trajectory cannot exit a polytope via a particular facet.

Lemma 2.3 ( Comparison principle [9] pg.102):

Consider the scalar differential equation

$$
\dot{w}=f(t, w), \quad w\left(t_{0}\right)=w_{0},
$$

where $f(t, w)$ is continuous in $t$ and locally Lipschitz in $w$, for all $t \geq 0$ and all $w \in \tilde{\mathcal{W}} \subset \mathbb{R}, \tilde{\mathcal{W}}$ a non-empty interval. Let $\left[t_{0}, T\right), T \leq \infty$, be the maximal interval of existence of the solution $w(t)$, and suppose $w(t) \in \tilde{\mathcal{W}}$ for all $t \in\left[t_{0}, T\right)$. Let $z(t)$ be a continuous function whose derivative satisfies the differential inequality

$$
\dot{z}(t) \leq f(t, z(t)), \quad z\left(t_{0}\right) \leq w_{0}
$$

with $z(t) \in \tilde{\mathcal{W}}$ for all $t \in\left[t_{0}, T\right)$. Then, $z(t) \leq w(t)$ for all $t \in\left[t_{0}, T\right)$.

The next result shows that if we are given a function $f$, which is locally Lipschitz, then the maximum of that function is also locally Lipschitz.

Lemma 2.4 ([3]): Given a function $f(x, y): \mathbb{R}^{n} \times \mathcal{Y} \rightarrow$ $\mathbb{R}$, with $\mathcal{Y}$ a compact metric space, and $f$ locally Lipschitz in $x$. Then $g(x):=\max _{y \in \mathcal{Y}} f(x, y)$ is also locally Lipschitz.

We are now ready to introduce an important result providing conditions for invariance of a facet, which was presented in [8] in a different way. A new proof is given, which provides an alternate view to the argument in [8].

Lemma 2.5: Consider the linear affine system $\dot{x}=A x+a$, $a \in \mathbb{R}^{n}$ defined on $P_{n}$. Suppose that for facets $F_{i}, i=1, \ldots k$ with normal vectors $h_{i}$, respectively, the following conditions hold:

$$
h_{i} \cdot \dot{x} \leq 0, \quad \forall x \in F_{i}, \quad i \in\{1, \ldots, k\} .
$$

Then all trajectories originating in $P_{n}$ that leave $P_{n}$ do so via an unrestricted facet $F_{j}, j \notin\{1, \ldots, k\}$.

Proof: Fix $i \in\{1, \ldots, k\}$ and w.l.o.g. assume that $F_{i}$ lies in the subspace $\left\{x \mid x_{n}=0\right\}$, so that $h_{i}$ is parallel to the $x_{n}$ axis. Also, assume that $\dot{x}=A x+a$ holds on the entire space $\mathbb{R}^{n}$, not only on $P_{n}$. We consider two cases: (1) a trajectory escaping via an interior point of the facet, and (2) a trajectory escaping via a boundary point.

Case 1. Let $\epsilon>0$. Consider the set $\mathcal{W}:=\left\{x \mid x_{n} \in\right.$ $\left.[0, \epsilon],\left(x_{1}, x_{2}, \ldots, x_{n-1}, 0\right) \in F_{i}\right\}$ (see Figure 1 ). We show that no trajectory can escape through an interior point of $F_{i}$. By way of contradiction, assume there exists a trajectory that leaves via the interior of $F_{i}$, i.e. there exists a time $t_{1}>0$ and some $\tau>0$ such that $x\left(t_{1}\right) \in F_{i}$, and $x\left(t_{1}+\tau\right) \notin F_{i}$ with $x_{n}\left(t_{1}+\tau\right) \in(0, \epsilon)$ (refer to Figure 1$)$. Note that if $h_{i} \cdot \dot{x}<0$ on $F_{i}$, by an elementary argument one can show that no trajectory can escape $P_{n}$ via $F_{i}$. So assume that at $x\left(t_{1}\right), h_{i} \cdot \dot{x}=\dot{x}_{n}=0$. We define two functions

$$
\begin{aligned}
f(x) & =\dot{x}_{n} \text { and } \\
g\left(x_{n}\right) & =\max _{\left\{\left(x_{1}, \ldots, x_{n-1}\right) \mid x \in \mathcal{W}\right\}} f(x) .
\end{aligned}
$$


By definition $\dot{x}_{n}=f(x) \leq g\left(x_{n}\right)$, for all $x \in \mathcal{W}$. Let $\dot{w}=g(w)$, with $w(0)=x_{n}\left(t_{1}\right)=0$. But $g(0)=0$ since $\dot{x}_{n} \leq 0$ on $F_{i}$. Therefore, $w(t)=0, \forall t \geq 0$, as $w$ is a scalar function. Now invoking the Comparison principle Lemma 2.3 with $\tilde{\mathcal{W}}=[0, \epsilon)$ and $g(w)$ locally Lipschitz by Lemma 2.4, we obtain that $x_{n}(t) \leq w(t)=0$ for all $t \in\left[t_{1}, t_{1}+\tau\right]$, a contradiction.

Case 2. We show that no trajectory can escape via a boundary point of $F_{i}$ without also escaping via $F_{k}$, which is unrestricted. As before, assume that at a boundary point $x\left(t_{1}\right)$ of $F_{i}, h_{i} \cdot \dot{x}=\dot{x}_{n}=0$. There are two possibilities: (a) there exists a facet $F_{j}, j \neq i$, with $x\left(t_{1}\right) \in F_{j}$ such that $h_{j} \cdot \dot{x}>0$, or (b) for all facets $F_{j}$ such that $x\left(t_{1}\right) \in F_{j}$, $h_{j} \cdot \dot{x} \leq 0$. In the first case, since $h_{j} \cdot \dot{x}>0$, then the trajectory exits $P_{n}$ via $F_{j}$. In the second case, we can use a similar proof as for an interior point, with a slight modification to our $\mathcal{W}$ set. Details are omitted.

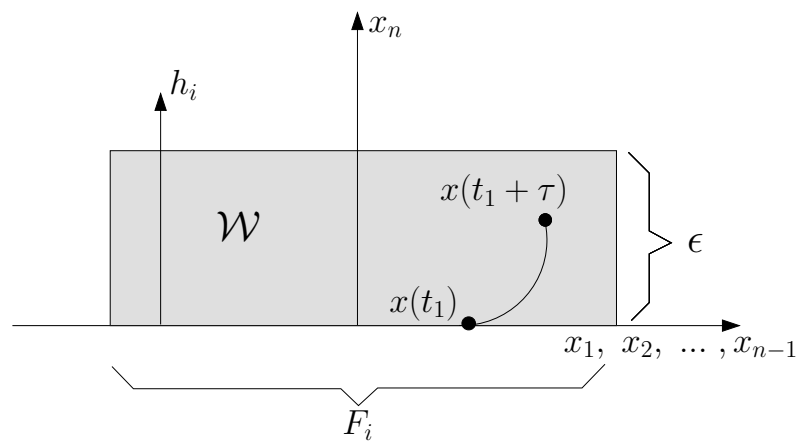

Fig. 1. Illustration for the proof of Lemma 2.5.

Observe that one can combine the results of Lemma 2.2 and of Lemma 2.5 to conclude that if there exists an input function guaranteeing that for each restricted facet $F_{i}$, the vector field at all vertices of $F_{i}$ satisfies the condition $h_{i} \cdot \dot{x} \leq$ 0 , then all trajectories which escape the polytope do so via an unrestricted facet.

\section{NECESSARY AND SUFFICIENT CONDITIONS}

This section derives the necessary and sufficient conditions for the general problem of reaching a set of facets of an ndimensional simplex in finite time, for a system evolving with linear affine dynamics.

The first result is central to the development of the necessary and sufficient conditions. It says that a system $\dot{x}=A x+a$ defined on a compact and convex set $P$ has no equilibria in $P$ if and only if there exists a vector $\xi \neq 0$ such that $\xi \cdot \dot{x}=\xi \cdot(A x+a)<0$ for all $x \in P$.

Theorem 3.1: Consider the linear affine system $\dot{x}=A x+$ $a$ with $x, a \in \mathbb{R}^{n}$, and a compact, convex set $P$. We have $A x+a \neq 0$ for all $x \in P$ if and only if there exists a $\xi \in \mathbb{R}^{n}$ such that $\xi \cdot \dot{x}=\xi \cdot(A x+a)<0$ for all $x \in P$.

\section{Proof:}

$(\Leftarrow) \quad$ Since $\xi \cdot(A x+a)<0$ for all $x \in P$, then clearly for all $x \in P, A x+a \neq 0$.

$(\Rightarrow \quad$ Since $P$ is compact and convex it follows that the image of $P$ under the map $x \mapsto A x+a$, denoted by $C_{1}=$ $A P+a$ is also compact and convex and does not contain the origin, by assumption. Thus, letting $C_{2}=\{0\}$ and using Theorem 2.1, there exists a hyperplane $H$ that separates $C_{1}$ and $C_{2}$ strongly. In other words, there exists $\epsilon>0$ and some $\xi \in \mathbb{R}^{n}$ such that for all $x \in P, \xi \cdot(A x+a) \leq-\epsilon$, or $\xi \cdot(A x+a)<0$.

A consequence of the above theorem is the following corollary, which ensures that all trajectories originating in a compact, convex set $P$ containing no equilibria eventually leave the set.

Corollary 3.1: Consider the system $\dot{x}=A x+a$, with $x, a \in \mathbb{R}^{n}$. Let $P \subseteq \mathbb{R}^{n}$ be compact and convex. Suppose that for all $x \in P, A x+a \neq 0$. Then, for each $x_{0} \in P$, the trajectory starting at $x_{0}$ eventually leaves $P$, i.e. $x\left(t_{1}\right) \notin P$ for some $t_{1}>0$.

Proof: From Theorem 3.1, we know that if for all $x \in$ $P, A x+a \neq 0$, then there exists a vector $\xi$ and a constant $\epsilon>$ 0 such that for all $x \in P, \xi \cdot(A x+a)>\epsilon$. Suppose $x(t)$ is a trajectory that lies in $P$ for all $t \geq 0$. Then $\xi \cdot(A x(t)+a)>\epsilon$ and using the Comparison principle Lemma 2.3, this implies $\xi \cdot(A x(t)+a)>\epsilon t$, for all $t \geq 0$. However, this contradicts the compactness of $P$.

The background results and the above observations lead to the first solution to Problem 1.1.

Theorem 3.2: Consider an affine system $\dot{x}=A x+B u+a$, with $x \in S_{n}$ and $u \in \mathbb{R}^{m}$. Problem 1.1 is solvable if and only if there exists a linear affine control $u$ with $u\left(v_{1}\right)=$ $u_{1}, \ldots, u\left(v_{n+1}\right)=u_{n+1}$ such that the closed loop system has no equilibria and the invariance conditions

$$
h_{i} \cdot\left(A v_{j}+B u_{j}+a\right) \leq 0 \quad j \in\{1, \ldots, n+1\}, i \in I_{v_{j}},
$$

are satisfied.

Proof:

$(\Rightarrow)$ It is obvious that the closed loop system will not have an equilibrium if Problem 1.1 is solved. For the proof of necessity of the invariance conditions, see [8].

$(\Leftarrow)$ By assumption, for the set of vertices $\left\{v_{1}, \ldots, v_{n+1}\right\}$ there exists a corresponding set of inputs $\left\{u_{1}, \ldots, u_{n+1}\right\}$. Invoking Lemma 2.1, there exists a linear affine control $u=$ $F x+g$, which guarantees that the desired input values are achieved at each vertex. Now we must show that the resultant input $u=F x+g$ solves Problem 1.1. First, by substituting for $u$, we obtain $\dot{x}=(A+F B) x+(B g+a)=\tilde{A} x+\tilde{a}$, and from the assumption that for all $x \in S_{n}, \tilde{A} x+\tilde{a} \neq 0$, Corollary 3.1 guarantees that all trajectories of this system will eventually leave $S_{n}$. That is, for each initial condition $x_{0} \in S_{n}$, there exists a time $t_{1}>0$ such that the trajectory starting at $x_{0}$ satisfies $x\left(t_{1}\right) \notin S_{n}$. Now it can be seen that this implies there exists $t_{0}<t_{1}$ and $\epsilon>0$ such that $x\left(t_{0}\right) \in$ $\partial S$ and $x(t) \notin S$ for all $t \in\left(t_{0}, t_{0}+\epsilon\right)$. For consider the set of times $\mathcal{T}=\left\{t \in\left[0, t_{1}\right): x(t) \in \partial S\right\}$ when $x(t)$ is in the boundary of $S$. Clearly $\mathcal{T}$ is nonempty and it is bounded. It is an elementary argument to show that $\mathcal{T}$ is also closed. Therefore $t_{0}:=\sup \mathcal{T}$ belongs to $\mathcal{T}$. Hence $x\left(t_{0}\right) \in \partial S$, $x\left(t_{1}\right) \notin S$, and it is easily seen that $t_{0}<t_{1}$. Let $\epsilon:=t_{1}-t_{0}$. Since $t_{0}$ is the last time $x(t)$ is in the boundary of $S$, we also 
have that $x(t) \notin S$ for all $t \in\left(t_{0}, t_{0}+\epsilon\right)$, as desired. Finally, using Lemma 2.2 and Lemma 2.5, the trajectory cannot leave via the restricted facets. This concludes the proof.

Theorem 3.2 gives conditions for the solvability of Problem 1.1. The invariance conditions agree with the results in [8], while the equilibrium condition introduces the missing link for solving the general problem of reaching a desired facet in finite time. However, if we were to construct an algorithm based on the conditions of Theorem 3.2, we would only be able to satisfy the invariance conditions and hope that the equilibrium condition would hold with the chosen controller. If not, then we would have to choose a different set of inputs $\left\{u_{1}^{\prime}, \ldots, u_{n+1}^{\prime}\right\}$ to construct a different input function $u^{\prime}=F^{\prime} x+g^{\prime}$ and check if the equilibrium lies outside $S_{n}$. In general this process does not give a guarantee that we can find a linear affine input function that solves the problem. However, from Lemma 3.1 we can replace the condition of no equilibria in a compact, convex set by the existence of a $\xi \in \mathbb{R}^{n}$ such that for all $x \in P$, $\xi \cdot(A x+a)<0$. Thus, with this observation a corollary to Theorem 3.2 is obtained that remedies the difficulty arising from checking the equilibrium condition.

Corollary 3.2: Consider the system $\dot{x}=A x+B u+a$, with $x \in S_{n}$. There exists a linear affine feedback $u=F x+$ $g$, with $F \in \mathbb{R}^{m \times n}$ and $g \in \mathbb{R}^{m}$, which solves Problem 1.1 if and only if there exists a set of inputs $u_{1}, \ldots, u_{n+1} \in \mathbb{R}^{m}$ and a vector $\xi$ such that the following hold:

1). Invariance Conditions: $h_{i} \cdot\left(A v_{j}+B u_{j}+a\right) \leq 0$

$$
j \in\{1,2, \ldots, n+1\}, i \in I_{v_{j}}
$$

\section{2). Flow Conditions: $\xi \cdot\left(A v_{j}+B u_{j}+a\right)<0$}

$$
j \in\{1,2, \ldots, n+1\} \text {. }
$$

Corollary 3.2 tells us that we only need to check several inequalities at the given vertices of the simplex. Moreover, if we know what the value of $\xi$ is, then the problem reduces to solving a set of linear inequalities. In fact, the sufficient conditions presented in [8] are a specific case of Corollary 3.2 , with $\xi$ set to $h_{1}$, and $I=\{2, \ldots, n+1\}$. It is clear that for our problem we don't have to restrict $\xi$ to be $h_{1}$. The examples at the end of the paper will illustrate several situations where setting $\xi$ to $h_{1}$ will not solve the general problem of leaving via a particular facet or set of facets, but a different $\xi$ will. The inequalities presented in the Corollary are still problematic, as the value of $\xi$ and the values for $u_{i}$ are unknown, making the feasibility of the inequalities a nonlinear problem. However, with the tools and algorithms presented in the literature on nonlinear programming (for instance [4] and references within), one can attempt to find the solution to the above inequalities. This nonlinear programming problem is beyond the scope of this paper and we leave the details to those interested.

\section{Algorithm}

In this section, we present an algorithm for finding an affine feedback controller that solves Problem 1.1. The algorithm parallels the steps outlined in [8], and is shown primarily for completeness.
Algorithm 4.1: We are given the linear affine system $\dot{x}=$ $A x+B u+a$ defined on an $n$-dimensional simplex $S_{n}$ with vertices $v_{i} \in V$, and the set $I$.

1) Check if the invariance and flow conditions of Corollary 3.2 can be satisfied, and the inputs $u_{1}, \ldots, u_{n+1}$ obtained. If not, the problem is unsolvable; otherwise go to the next step.

2) Solve (6) for $F$ and $g$ and construct the linear affine controller $u=F x+g$.

The drawback of the algorithm, as previously mentioned, is that we need to solve nonlinear inequalities in Step 1. However, from Theorem 3.2 we know that if we satisfy the invariance conditions and find the input $u=F x+g$, such that the closed-loop system has no equilibria, then Problem 1.1 is solved. This method may not always yield the desired input, but as will be illustrated in the next section, it can avoid the effort of finding a feasible solution to the nonlinear inequalities of step one.

\section{EXAMPLES}

To illustrate the results of the previous sections two examples are given. In both examples the conditions presented in [8] fail, but reachability of the desired facet is still achieved.

Example 5.1: Consider the system

$$
\dot{x}=\left[\begin{array}{ll}
0 & 1 \\
0 & 0
\end{array}\right] x+\left[\begin{array}{l}
0 \\
1
\end{array}\right] u+\left[\begin{array}{l}
4 \\
1
\end{array}\right]
$$

defined on a simplex $S_{2}$ with vertices $v_{1}=(-1,-3), v_{2}=$ $(4,-1)$ and $v_{3}=(3,-6)$, and normals $h_{1}=[5-1], h_{2}=$ $\left[\begin{array}{ll}-3 & -4\end{array}\right]$ and $h_{3}=\left[\begin{array}{ll}-2 & 5\end{array}\right]$. We require that all trajectories originating in $S_{2}$ leave via $F_{1}$. The conditions in [8] on $v_{3}$ are

1) $h_{1}^{T} B u_{3}>-h_{1}^{T}\left(A v_{3}+a\right) \Rightarrow u_{3}<-11$ $h_{2}^{T} B u_{3} \leq-h_{2}^{T}\left(A v_{3}+a\right) \Rightarrow u_{3} \geq \frac{1}{2}$

Clearly the conditions on vertex $v_{3}$ fail. However, by checking the invariance conditions of Theorem 3.2, and ensuring there is no equilibrium for the closed loop system inside $S_{2}$, we notice that reaching $F_{1}$ without leaving through any other facet is possible. The conditions for restricting the two facets result in the satisfaction of the four conditions below

1) $h_{3}^{T} B u_{2} \leq-h_{3}^{T}\left(A v_{2}+a\right) \Rightarrow u_{2} \leq 0.2$ $h_{3}^{T} B u_{1} \leq-h_{3}^{T}\left(A v_{1}+a\right) \Rightarrow u_{1} \leq-0.6$

2) $h_{2}^{T} B u_{1} \leq-h_{2}^{T}\left(A v_{1}+a\right) \Rightarrow u_{1} \geq-1.75$ $h_{2}^{T} B u_{3} \leq-h_{2}^{T}\left(A v_{3}+a\right) \Rightarrow u_{3} \geq 0.5$.

With the arbitrary choice of $u_{1}=-1.175, u_{2}=0.2$ and $u_{3}=0.5$, we can construct the linear affine controller $u=F x+g$, with $F=\left[\begin{array}{ll}0.325-0.125\end{array}\right]$ and $g=-1.225$, which results in the overall closed loop system having an equilibrium at approximately [-0.8462 4.0]. With this controller we are guaranteed not to exit through $F_{2}$ or $F_{3}$ since the invariance conditions hold; moreover, by the results of the previous sections we are guaranteed to leave the simplex via $F_{1}$, i.e. $I=\{2,3\}$. The phase portrait of the closed loop system is illustrated in Figure 2.

Example 5.2: Consider the system

$$
\dot{x}=\left[\begin{array}{ccc}
1 & 2 & 0 \\
0 & -1 & 0 \\
0 & 0 & 1
\end{array}\right] x+\left[\begin{array}{l}
1 \\
1 \\
0
\end{array}\right] u+\left[\begin{array}{l}
2 \\
3 \\
1
\end{array}\right]
$$


defined on a simplex $S_{3}$ with vertices $v_{1}=(0,0,0)$, $v_{2}=(1,2,0), v_{3}=(-2,-1,0)$, and $v_{4}=(0,0,1)$, and corresponding normals $h_{1}=\left[\begin{array}{lll}-3 & 3 & 3\end{array}\right], h_{2}=\left[\begin{array}{lll}1 & -2 & 0\end{array}\right]$, $h_{3}=\left[\begin{array}{lll}2 & -1 & 0\end{array}\right]$, and $h_{4}=\left[\begin{array}{lll}0 & 0 & -1\end{array}\right]$. We require that all trajectories originating in $S_{3}$ leave via $F_{1}$, i.e. $I=\{2,3,4\}$. Once again, by verifying the condition presented in [8] on $v_{2}$ we must satisfy

$$
\text { 1) } h_{1}^{T} B u_{2}>-n_{1}^{T}\left(A v_{2}+a\right) \text {, implying that } 0>15
$$

which is infeasible. However, the invariance conditions imply that $-4 \leq u_{1} \leq-1, u_{2} \leq-13, u_{3} \geq-10$ and $-4 \leq u_{4} \leq$ -1 . Choosing $u_{1}=-2, u_{2}=-15, u_{3}=12$ and $u_{4}=-2$ we can construct the linear affine controller $u=F x+g$, with $F=\left[\begin{array}{ll}-5 & -40\end{array}\right]$ and $g=-2$. The closed loop system has an equilibrium at $(-0.2,0.4,-1)$. Since all the facets have been restricted and the equilibrium lies outside of $S_{3}$, we can guarantee all trajectories leave through the desired facet

The reason that the conditions presented in [8] failed in these examples is because $\xi$ cannot be set to $h_{1}$ in Corollary 3.2. Of course, it should be noted that in [8] the goal was not to solve the general problem of leaving via a particular facet, but to solve a specific problem with all trajectories flowing in a particular direction with respect to $h_{1}$.

\section{Conclusion}

The results presented in this paper have been heavily influenced by the work of Habets and van Schuppen [8]. We have presented a generalization of their problem by considering an arbitrary number of restricted facets and by dropping the restriction that $t_{0}$ in Problem 1.1 must be the first time instant at which the state reaches the exit facet. The results illustrate that through a test on the vertices of the polytope one can derive a controller that restricts the flow of the system. Moreover, by checking that the closedloop system contains no equilibria, we have shown that the system will always leave through the desired facet in finite time. The results presented thus bridge the gap with the work of [8], showing that the necessary and sufficient conditions presented in [8] are too restrictive if reachability of a facet is the only goal. Finally, the examples illustrate the simplicity

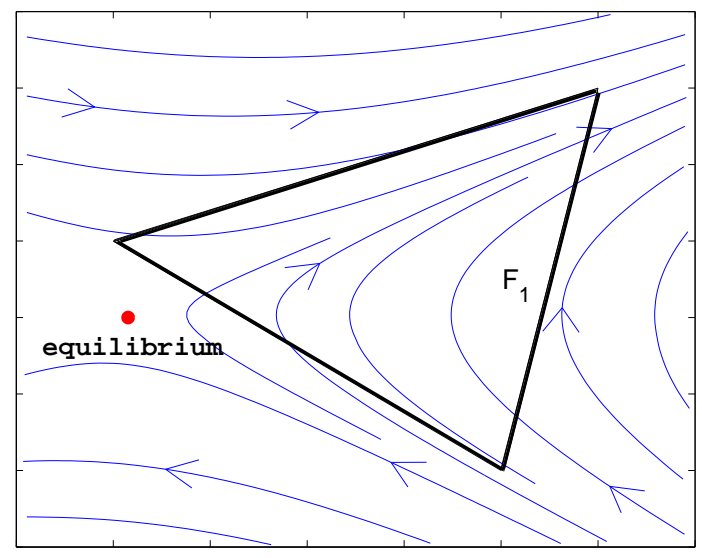

Fig. 2. Phase portrait of the closed loop system for Example 5.1. and usefulness of the results presented in the paper. In both situations an arbitrary choice of the input resulted in an adequate controller; namely, a controller that enforces the equilibrium to lie outside of the simplex. In general, one might not be able to make an arbitrary choice for the control, and instead one must work with the nonlinear constraints given by Corollary 3.2.

With convexity and linearity of the problem in mind, the authors are currently pursuing the idea of transforming the nonlinear conditions into linear ones by finding a legitimate $\xi$.

\section{ACKNOWLEDGEMENTS}

The first author thanks Lin Zhiyun, PhD candidate in the System Control Group, University of Toronto, for his collaboration on the proof of Lemma 2.5.

\section{REFERENCES}

[1] R. Alur and G.J. Pappas, editors. Hybrid Systems: Computation and Control, Lecture Notes in Computer Science (HSCC'04), Volume 2993, Springer-Verlag, Philadelphia, 2004.

[2] V.I. Arnold. Ordinary Differential Equations, The MIT Press, Fourth Printing, Cambridge, Massachusetts 1985.

[3] C. Berge and A. Ghouila-Houri. Programming, Games, and Transportation Networks, John Wiley and Sons, 1965.

[4] D.P. Bertsekas. Nonlinear Programming 2nd edition, Athena Scientific, Belmont Massachusetts 1999.

[5] F. Blanchini. Set invariance in control (Survey paper), Automatica, 35 (1999) 1747-1767.

[6] E.B. Castelan and J.C. Hennet. On Invariant Polyhedra of ContinuousTime Linear Systems, IEEE Transactions on Automatic Control, 38 (1993), 1680-1685.

[7] L.C.G.J.M. Habets and J.H. van Schuppen. Control of piecewise-linear hybrid systems on simplices and rectangles, in: M.D. Di Benedetto and A.L. Sangiovanni-Vincentelli (Eds.) Hybrid Systems: Computation and Control, Lecture Notes in Computer Science, Springer Verlag 2001, Volume 2034 261-274.

[8] L.C.G.J.M. Habets and J.H. van Schuppen. A control problem for affine dynamical systems on a full-dimensional polytope, Automatica 40:21-35 2004.

[9] H.K. Khalil. Nonlinear Systems, Prentice Hall, New Jersey 2002.

[10] K.K. Lee and A. Arapostathis. On the controllability of piecewiselinear hypersurface systems, Systems \& Control Letters, 9 (1987) 8996.

[11] O. Maler and A. Pnueli, editors. Hybrid Systems: Computation and Control, Lecture Notes in Computer Science (HSCC'03), Volume 2623, Springer-Verlag, Prague 1999.

[12] R.T Rockafellar. Convex Analysis, Princeton University - Princeton, New Jersey 1970.

[13] E.D. Sontag. Nonlinear regulation: The piecewise linear approach, IEEE Trans. Automatic Control 26:346-358, 1981.

[14] E.D. Sontag. Remarks on piecewise-linear algebra, Pacific Journal of Math, 98:183-201, 1982.

[15] E.D. Sontag, Interconnected automata and linear systems: A theoretical framework in discrete-time, in: R. Alur and T.A. Henzinger (Eds.) Hybrid Systems III: Verification and Control, Lecture Notes in Computer Science, Springer Verlag, Vol 1066 436-448, 1996.

[16] M. Vassilaki and G. Bitsoris, Constrained regulation of linear continuous-time dynamical systems, Systems \& Control Letters, 13 (1989), 247-252.

[17] V.M. Veliov and M. I. Krastanov, Controllability of piecewise linear systems, Systems \& Control Letters, 7 (1986), 335-341. 